Article

\title{
Nutritional Value and Volatile Compounds of Black Cherry (Prunus serotina) Seeds
}

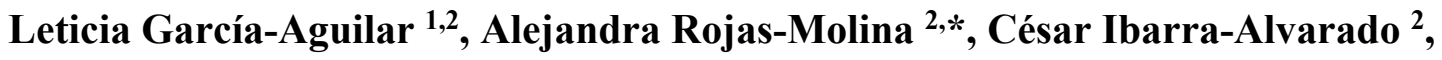 \\ Juana I. Rojas-Molina ${ }^{2}$, Pedro A. Vázquez-Landaverde ${ }^{3}$, Francisco J. Luna-Vázquez ${ }^{2}$ \\ and Miguel A. Zavala-Sánchez ${ }^{4}$
}

1 Doctorado en Ciencias Biológicas y de la Salud, Universidad Autónoma Metropolitana, Unidad Xochimilco, Mexico, D.F. 04960, Mexico; E-Mail: laetitia_spp@yahoo.com

2 Laboratorio de Investigación Química y Farmacológica de Productos Naturales, Facultad de Química, Universidad Autónoma de Querétaro, Querétaro, Qro. 76010, Mexico; E-Mails: cibarra@uaq.mx (C.I.-A.); jirojasmolina@gmail.com (J.I.R.-M.); fjlunavz@yahoo.com.mx (F.J.L.-V.)

3 Centro de Investigación en Ciencia Aplicada y Tecnología Avanzada del Instituto Politécnico Nacional, Unidad Querétaro. Cerro Blanco No. 141. Col. Colinas del Cimatario, Querétaro, Qro.76090, Mexico; E-Mail: pavazquez@ipn.mx

4 Departamento de Sistemas Biológicos, Universidad Autónoma Metropolitana, Unidad Xochimilco, México, D.F. A.P. 23-181, Mexico; E-Mail: mzavala@correo.xoc.uam.mx

* Author to whom correspondence should be addressed; E-Mail address: rojasa@uaq.mx; Tel.: +52-442-192-1200 (ext. 5527).

Academic Editor: Luca Forti

Received: 4 January 2015 / Accepted: 11 February 2015 / Published: 17 February 2015

\begin{abstract}
Prunus serotina (black cherry), commonly known in Mexico as capulín, is used in Mexican traditional medicine for the treatment of cardiovascular, respiratory, and gastrointestinal diseases. Particularly, P. serotina seeds, consumed in Mexico as snacks, are used for treating cough. In the present study, nutritional and volatile analyses of black cherry seeds were carried out to determine their nutraceutical potential. Proximate analysis indicated that P. serotina raw and toasted seeds contain mostly fat, followed by protein, fiber, carbohydrates, and ash. The potassium content in black cherry raw and toasted seeds is high, and their protein digestibility-corrected amino acid scores suggest that they might represent a complementary source of proteins. Solid phase microextraction and gas chromatography/flame ionization detection/mass spectrometry analysis allowed identification of
\end{abstract}


59 and 99 volatile compounds in the raw and toasted seeds, respectively. The major volatile compounds identified in raw and toasted seeds were 2,3-butanediol and benzaldehyde, which contribute to the flavor and odor of the toasted seeds. Moreover, it has been previously demonstrated that benzaldehyde possesses a significant vasodilator effect, therefore, the presence of this compound along with oleic, linoleic, and $\alpha$-eleostearic fatty acids indicate that black cherry seeds consumption might have beneficial effects on the cardiovascular system.

Keywords: Prunus serotina seeds; black cherry seeds; volatile compounds; proximal analysis; amino acid profile

\section{Introduction}

Prunus serotina Ehrh (American black cherry), commonly called "capulín" in Mexico, is a native North American tree that belongs to the family Rosaceae [1]. It grows wildly or under cultivated conditions in Mexican highlands and in some regions of Guatemala, Colombia, and Venezuela [2]. In recent times, it has spread widely in some European countries, like Belgium, the Netherlands, and Germany [3]. P. serotina is a very fast-growing tree which has an oval silhouette, its low branches normally droop and touch the ground, the leaves are dark green and shiny; the fruit is a little juice drupe about $1 \mathrm{~cm}$ long with a stone which contains a seed [4].

In Mexico, P. serotina has been used since colonial times for nourishment and medicinal purposes. The fruit is commonly consumed fresh, as jam, liquors or syrups, and the seeds are consumed roasted and salted as snacks [2]. In Mexican traditional medicine, teas and syrups prepared from the leaves and fruits are highly appreciated for treating hypertension, stomach upsets, mouth infections, diarrhea, malaria, bronchitis, and cough [5,6], and the seeds are used in decoction for the treatment of cough [5]. Black cherry bark has been used by the Iroquois, Ojibwa, Malecite, and Delaware indigenous people from the North American boreal forest regions of Canada for treating diabetes-related symptoms [7].

Recently, our research group carried out chemical and pharmacological studies of the leaves, fruits and seeds of $P$. serotine. A bio-directed phytochemical study of the methanolic extract of the leaves led to the isolation of hyperoside and ursolic acid as the main vasodilator compounds. It was also found that benzyl alcohol, benzaldehyde, cinnamyl alcohol, and cinnamaldehyde were the major constituents of the essential oil obtained from the leaves, these compounds promoted vascular smooth muscle relaxation [8]. Regarding $P$. serotina fruits, we found that they have a high content of phenolic compounds such as chlorogenic acid, gallic acid, caffeic acid, catechin, epicatechin, quercetin, and kaempferol glycosides, which are directly related to the high antioxidant capacity and significant vasodilatory effect of the aqueous extract of the fruits [9]. Additionally, physicochemical and chemical characterization of black cherry seed oil demonstrated that it is mainly composed of polyunsaturated fatty acids, including oleic, linoleic, and $\alpha$-eleostearic acids [10]. These results support the potential therapeutic significance of $P$. serotina seed oil, since it has been extensively demonstrated that consumption of unsaturated fatty acids reduce plasma lipids and reduce atherogenesis by decreasing inflammation of macrophages and vascular endothelial cells [11,12]. Moreover, it has been reported that $\alpha$-eleostearic acid is effective in 
suppressing growth in cancer cells, and it has been proposed as a chemotherapeutic agent against breast cancer [13].

Although black cherry seeds contain cyanogenic glycosides [14], there are no reports of human intoxication related to their intake, since they are consumed toasted, which indicates that the toasting process decreases the content of these compounds [15]. Heat treatment also provokes emission of volatile compounds contained in the seeds, which undoubtedly contribute to their pleasant and characteristic flavor. These volatile components might also possess pharmacological activity [16].

Currently, the nutritional value of black cherry seeds and their volatile components have not been investigated. Therefore, in the present study the proximate composition, vitamin and mineral content of these seeds were determined in order to assess their nutritional value. In addition, the volatile compounds present in the seeds were analyzed by gas chromatography coupled to mass spectrometry.

\section{Results and Discussion}

\subsection{Proximate Composition}

Proximate compositions of raw and toasted black cherry seeds, Prunus dulcis (almonds) and Arachis hypogaea (peanuts) are shown in Table 1. There were no significant differences between protein, ash, crude fiber or carbohydrate contents of the raw and roasted seeds, suggesting that the toasting process does not affect the proximate composition. The mean values of protein content were $37.95 \% \pm 0.16 \%$ and $36.55 \% \pm 0.22 \%$ for raw and toasted seeds, respectively. These values were significantly higher than those of almonds (19.91\%), and peanuts $(22.82 \%)$, whose protein content is consistent with earlier reports $[17,18]$. P. serotine seeds possess a protein content which is comparable to that of seeds of other Prunus species, including apricots ( $P$. armeniaca L., 37.4\%), sweet cherries (P. avium L. 31.7\%), sour cherries (P. cerasus L., 31.7\%), nectarines (P. persica ar. nectarina (Aiton) Maxim, 38.7\%), peaches (P. persica (L.) Batsch var. persica, 33.4\%), and plums (P. domestica L., 35.9\%) [19].

Table 1. Proximate composition of raw and toasted black cherry seeds, almond, and peanut.

\begin{tabular}{ccccc}
\hline $\begin{array}{c}\text { Component } \\
\text { (\% Dry Basis) }\end{array}$ & $\begin{array}{c}\text { Raw Black } \\
\text { Cherry Seeds }\end{array}$ & $\begin{array}{c}\text { Toasted Black } \\
\text { Cherry Seeds }\end{array}$ & Almonds & Peanuts \\
\hline Moisture & $8.92 \pm 0.42^{\mathrm{a}}$ & $10.75 \pm 0.35^{\mathrm{a}}$ & $6.08 \pm 0.4^{\mathrm{b}}$ & $5.45 \pm 0.38^{\mathrm{c}}$ \\
Fat & $40.37 \pm 0.73^{\mathrm{a}}$ & $39.97 \pm 0.20^{\mathrm{a}}$ & $49.64 \pm 0.42^{\mathrm{b}}$ & $41.12 \pm 1.51^{\mathrm{a}}$ \\
Protein & $37.95 \pm 0.16^{\mathrm{a}}$ & $36.55 \pm 0.22^{\mathrm{a}}$ & $19.91 \pm 0.01^{\mathrm{b}}$ & $22.82 \pm 0.01^{\mathrm{c}}$ \\
Ash & $3.19 \pm 0.18^{\mathrm{a}}$ & $2.72 \pm 0.21^{\mathrm{a}}$ & $3.18 \pm 0.21^{\mathrm{a}}$ & $2.41 \pm 0.19^{\mathrm{a}}$ \\
Crude Fiber & $10.73 \pm 1.49^{\mathrm{a}}$ & $12.12 \pm 4.06^{\mathrm{a}}$ & $10.91 \pm 1.45^{\mathrm{a}}$ & $9.21 \pm 1.59^{\mathrm{a}}$ \\
Carbohydrates & $7.76 \pm 2.24^{\mathrm{a}}$ & $8.65 \pm 4.28^{\mathrm{a}}$ & $10.26 \pm 1.98^{\mathrm{b}}$ & $18.95 \pm 2.68^{\mathrm{c}}$ \\
\hline
\end{tabular}

Notes: Data are given as mean \pm standard deviation $(n=3$ independent experiments performed in different samples). a, b and c: Values in the same row followed by the same superscript letter are not significantly different $(p>0.05)$.

Lipid content of $P$. serotina raw $(40.37 \% \pm 0.73 \%)$ and toasted seeds $(39.97 \% \pm 0.20 \%)$ was not significantly different from that of peanuts $(41.12 \% \pm 1.51 \%)$. However, it was significantly lower than almonds lipid content $(49.64 \% \pm 0.42 \%)$. It is well known that seed oils are mainly composed by mono- and polyunsaturated fatty acids, thus oleic (61\%) and linoleic (29\%) acids are the main fatty acids 
contained in almond; oleic acid (81\%) is abundant in peanut oil [20], whereas, black cherry seed oil is rich in oleic (35\%), linoleic (27\%) and $\alpha$-eleostearic (27\%) acids [10].

These findings indicate that $P$. serotina edible seeds are a good source of unsatured fatty acids with potential health benefits [21]. The mean values of ash and crude fiber of raw and toasted $P$. serotina seeds, almonds and peanuts did not differ significantly. The crude fiber content was similar to that of other seeds and nuts that represent a complementary source of fiber, like hazelnut ( $9 \%-13 \%)$ or pumpkin seeds $(12.1 \%)[22,23]$. Adequate intake fiber is related to the obesity prevention and better glycemic control in patients with type 2 diabetes mellitus [24,25].

\subsection{Minerals}

The mineral composition ( $\mathrm{Ca}, \mathrm{Fe}, \mathrm{Mg}, \mathrm{P}, \mathrm{K}, \mathrm{Zn}$ and $\mathrm{Na}$ ) of raw and toasted black cherry seeds, almonds and peanuts are presented in Table 2. The $\mathrm{Mg}$ content of raw and toasted black cherry seeds was unaffected by heat processing, but the $\mathrm{Ca}, \mathrm{Fe}, \mathrm{P}, \mathrm{K}, \mathrm{Zn}$, and $\mathrm{Na}$ content diminished after the toasting process. Usually, nuts and seeds are consumed toasted and salted, and toasting might have an important influence on their nutrimental quality. Chitra et al. [26] and Gamel et al. [27] have pointed out that Ca and Fe content significantly reduced after toasting or autoclaving seeds of soybean and pigeon peas.

Table 2. Mineral composition of raw and toasted black cherry seeds, almond and peanut.

\begin{tabular}{ccccc}
\hline $\begin{array}{c}\text { Mineral (Dry Basis) } \\
\text { mg/100 g }\end{array}$ & $\begin{array}{c}\text { Raw Black } \\
\text { Cherry Seeds }\end{array}$ & $\begin{array}{c}\text { Toasted Black } \\
\text { Cherry Seeds }\end{array}$ & Almonds & Peanuts \\
\hline $\mathbf{C a}$ & $192.30 \pm 0.58^{\mathrm{a}}$ & $127.11 \pm 17.51^{\mathrm{b}}$ & $305.43 \pm 1.88^{\mathrm{c}}$ & $91.43 \pm 0.05^{\mathrm{d}}$ \\
$\mathbf{F e}$ & $9.49 \pm 0.3^{\mathrm{a}}$ & $1.21 \pm 0.003^{\mathrm{b}}$ & $6.08 \pm 0.01^{\mathrm{c}}$ & $8.31 \pm 0.02^{\mathrm{d}}$ \\
$\mathbf{M g}$ & $249.15 \pm 0.34^{\mathrm{a}}$ & $216.68 \pm 18.75^{\mathrm{a}}$ & $282.09 \pm 0.32^{\mathrm{b}}$ & $172.75 \pm 0.77^{\mathrm{c}}$ \\
$\mathbf{P}$ & $439.0 \pm 0.16^{\mathrm{a}}$ & $323.40 \pm 0.14^{\mathrm{b}}$ & $387.03 \pm 0.7^{\mathrm{c}}$ & $347.41 \pm 0.07^{\mathrm{d}}$ \\
$\mathbf{K}$ & $873.22 \pm 12.64^{\mathrm{a}}$ & $454.82 \pm 0.41^{\mathrm{b}}$ & $656.25 \pm 23.80^{\mathrm{c}}$ & $571.57 \pm 10.03^{\mathrm{d}}$ \\
$\mathbf{Z n}$ & $3.40 \pm 0.10^{\mathrm{a}}$ & $2.96 \pm 0.24^{\mathrm{b}}$ & $4.48 \pm 0.17^{\mathrm{c}}$ & $3.92 \pm 0.10^{\mathrm{d}}$ \\
$\mathbf{N a}$ & $82.98 \pm 0.90^{\mathrm{a}}$ & $23.59 \pm 0.8^{\mathrm{b}}$ & $76.57 \pm 0.38^{\mathrm{a}}$ & $62.99 \pm 0.65^{\mathrm{c}}$ \\
\hline
\end{tabular}

Notes: Data are given as mean \pm standard deviation $(n=3$ independent experiments performed in different samples). a, b, c and d: Values in the same row followed by the same superscript letter are not significantly different $(p>0.05)$.

Raw black cherry seeds possess a higher content of $\mathrm{Ca}, \mathrm{Fe}, \mathrm{Mg}, \mathrm{K}$ and $\mathrm{Na}$ than that found in peanuts. On the other hand, $\mathrm{Fe}, \mathrm{K}$, and $\mathrm{Na}$ content is greater than that of almonds. Interestingly, the $\mathrm{K}$ content in black cherry seeds $(873.22 \pm 12.64 \mathrm{mg} / 100 \mathrm{~g})$ is significantly higher than that of almonds and peanuts, suggesting that $P$. serotina seeds represent a good complementary source of this mineral.

\subsection{Vitamins}

Vitamin analysis showed that raw and toasted P. serotina seeds do not contain vitamins A and C. Vitamin E or $\alpha$-tocopherol was detected at a concentration of $3.916 \mathrm{mg} / 100 \mathrm{~g}$ in raw seeds, while in toasted seed it was absent. This lost may be associated with vitamin degradation due to temperature exposure [27]. Our results are in agreement with previous studies carried out on other seeds and nuts, such as pumpkin seeds, walnuts and peanuts, which do not contain detectable values of vitamins A and 
$\mathrm{C}[28,29]$. Vitamin E content of black cherry seeds is similar to that of pine nuts $(4.1 \mathrm{mg} / 100 \mathrm{~g})$ and peanuts $(6.1 \mathrm{mg} / 100 \mathrm{~g})$, however it is lower than that of almonds and hazelnuts $(24.2 \mathrm{mg} / 100 \mathrm{~g}$ and $31.4 \mathrm{mg} / 100 \mathrm{~g}$, respectively) [28].

\subsection{Protein Nutritional Quality}

Since black cherry seeds contain higher levels of protein $(37.95 \% \pm 0.16 \%)$ than other seeds, their protein nutritional quality was assessed. Amino acid composition data are presented in Table 3 . It is important to mention that in this experiment tryptophan was not determined.

Table 3. Protein amino acids composition of raw and toasted black cherry seeds.

\begin{tabular}{ccc}
\hline $\begin{array}{c}\text { Amino } \\
\text { Acid }\end{array}$ & $\begin{array}{c}\text { Raw Seeds mg/g } \\
\text { Protein }\end{array}$ & $\begin{array}{c}\text { Toasted Seeds mg/g } \\
\text { Protein }\end{array}$ \\
\hline Asp & 112.29 & 116.97 \\
Glu & 256.84 & 273.73 \\
Ser & 32.84 & 42.11 \\
His & 21.60 & 21.29 \\
Gly & 37.43 & 38.82 \\
Thr & 52.85 & 59.16 \\
Arg & 84.24 & 87.42 \\
Ala & 41.47 & 44.06 \\
Tyr & 48.75 & 60.99 \\
Met & 8.93 & 9.83 \\
Val & 45.48 & 45.62 \\
Phe & 48.64 & 52.00 \\
Ile & 39.17 & 40.33 \\
Leu & 75.10 & 82.11 \\
Lys & 8.85 & 11.17 \\
\hline
\end{tabular}

Another protein quality measure is digestibility. Differences among the in vitro protein digestibility values of raw and toasted black cherry seeds were not significant $(88.12 \% \pm 0.72 \%$ and $89.40 \% \pm 1.32 \%$, respectively). These values were similar to that of almonds $(90.15 \% \pm 0.85 \%)$, however they were lower than those of peanuts $(94.06 \% \pm 0.78 \%)$ and casein $(98.51 \% \pm 0.82 \%)$, which was used as control. It has been reported that in vitro protein digestibility values greater than $80 \%$ are related to an efficient amino acid bioavailability [27]. Therefore, these results suggest that black cherry seed proteins are highly bioavailable.

Considering these findings, the protein digestibility-corrected amino acid score (PDCAAS) was determined. This method is accepted as the most recognized approach for assessing the protein quality of foods [30]. Every essential amino acid receives a score, but the final PDCAAS value corresponds to that of the limiting amino acid. The highest PDCAAS value for a given protein is 1.0, which indicates that a protein provides adequate amounts of all the essential amino acids [31]. Table 4 shows that lysine is the limiting amino acid in P. serotina seeds, therefore, PDCAAS values for raw and toasted black cherry seeds protein are 0.13 and 0.18 , respectively. These values are lower than those reported for 
almonds (0.23) [17] and peanuts (0.69) [32]. These results suggest that black cherry seeds could be a complementary source of proteins.

Table 4. Black cherry seeds amino acid score (AAS).

\begin{tabular}{cccc}
\hline \multirow{2}{*}{ AA } & $\begin{array}{c}\text { FAO Reference } \\
\text { Pattern }\end{array}$ & AAS $*$ & AAS * \\
\cline { 3 - 4 } & 19 & Raw Seeds & Toasted Seeds \\
\hline His & 34 & 1.37 & 1.12 \\
Thr & 25 & 1.55 & 1.74 \\
Met & 35 & 0.36 & 0.39 \\
Val & 63 & 1.30 & 1.33 \\
Phe & 28 & 0.77 & 0.82 \\
Ile & 66 & 1.40 & 1.44 \\
Leu & 58 & 1.38 & 1.24 \\
Lys ** & 0.15 & 0.19 \\
\hline
\end{tabular}

Notes: * Amino acid score $(\mathrm{AAS})=\mathrm{AA}$ content of test protein/reference AA pattern, where reference AA pattern is the amino acid requirement for a preschool child (2-5 years) [30].

** Limiting amino acid.

\subsection{Volatile Compounds}

Volatile compounds of raw and toasted black cherry seeds were extracted by head space solid phase micro extraction and analyzed by gas chromatography-mass spectrometry, using DB-5 and Wax capillary columns in order to detect a wide range of polar and non-polar compounds. A total of 59 and 99 volatile compounds were identified in the raw and toasted P. serotina seeds, respectively (Table 5). The identified volatile compounds comprise aldehydes, alcohols, ketones, carboxylic acids, esters, hydrocarbons, and pyrazines.

The predominant aldehyde identified in raw and toasted seeds was benzaldehyde. This compound has a characteristic pleasant almond-taste and aroma [33] and has been identified in bitter and sweet almonds [34], hazelnuts [33], and seeds of other species of the genus Prunus [35,36]. Benzaldehyde is produced by enzymatic degradation of the cyanogenic glycosides amygdalin and prunasin [35,37,38]. Considering on one hand that benzaldehyde content in toasted seeds was higher than that in raw seeds, and on the other, that bitterness attributed to the presence of amygdalin [39,40] diminished in toasted seeds, it is very likely that the toasting process may induce benzaldehyde formation from cyanogenic glycosides. Benzaldehyde might also be generated from phenylalanine during heating processes $[41,42]$ as was demonstrated by Alasalvar et al. [33] in hazelnuts, pumpkin seeds [43], and peanuts [44]. It is worth noting that we have previously identified benzaldehyde as one of the major components in black cherry leaves essential oil, we also proved that this compound induces a significant concentration-dependent vasodilator effect [8]. Therefore, the presence of this compound along with oleic, linoleic, and $\alpha$-eleostearic fatty acids indicate that black cherry seeds consumption might have beneficial effects on the cardiovascular system. 
Table 5. Volatile compounds on raw and toasted black cherry seeds.

\begin{tabular}{|c|c|c|c|c|c|c|c|}
\hline \multicolumn{2}{|c|}{$\begin{array}{c}\text { RI } \\
\text { Calculated }^{\text {a }}\end{array}$} & \multicolumn{2}{|c|}{$\begin{array}{c}\text { RI } \\
\text { Literature }^{b}\end{array}$} & \multirow[t]{2}{*}{ Compound } & \multirow{2}{*}{$\begin{array}{l}\operatorname{Raw}^{c} \\
\left(\operatorname{ppm}^{\mathrm{d}}\right)\end{array}$} & \multirow{2}{*}{$\begin{array}{c}\text { Toasted }^{\mathrm{c}} \\
\left(\mathrm{ppm}^{\mathrm{d}}\right) \\
\end{array}$} & \multirow[t]{2}{*}{ ID $^{\mathrm{e}}$} \\
\hline DB5 & WAX & DB5 & WAX & & & & \\
\hline \multicolumn{8}{|c|}{ Aldehydes } \\
\hline 573 & 805 & & 801 & Propanal, 2-methyl- & & 0.604 & MS, RI \\
\hline 638 & & 640 & & 2-Butenal & & 0.180 & MS, RI \\
\hline 642 & 908 & 642 & & Butanal, 3-methyl- & & 1.095 & MS, RI \\
\hline 652 & 910 & 653 & & Butanal, 2-methyl- & & 1.419 & MS, RI \\
\hline 695 & 965 & 695 & 970 & Pentanal & & 2.065 & MS, RI \\
\hline \multirow[t]{2}{*}{740} & & 740 & & 2-Butenal, 2-methyl- & & 0.500 & MS, RI \\
\hline & 1074 & & 1078 & Hexanal & & 0.096 & MS, RI \\
\hline 834 & 1440 & 835 & 1443 & Furfural & & 2.609 & MS, RI \\
\hline 902 & & 905 & & Heptanal & & 0.084 & MS, RI \\
\hline 908 & & 909 & & Propanal, 3-(methylthio)- & & 0.041 & MS, RI \\
\hline & 1199 & & 1196 & 2-Hexenal, $(E)-$ & & 0.014 & MS, RI \\
\hline 964 & & 963 & & 2-Furancarboxaldehyde, 5-methyl- & & 0.112 & MS, RI \\
\hline 967 & 1508 & 969 & 1508 & Benzaldehyde & 1.371 & 7.597 & MS, RI \\
\hline 1053 & & 1053 & & Benzeneacetaldehyde & & 0.411 & MS, RI \\
\hline \multirow[t]{2}{*}{1106} & & 1108 & & Nonanal & 0.056 & 0.201 & MS, RI \\
\hline & 1443 & & 1455 & 3-Furaldehyde & 0.090 & & MS, RI \\
\hline 1221 & 1697 & 1221 & 1697 & 2,4-Nonadienal, $(E, E)$ - & & 0.091 & MS, RI \\
\hline 1279 & 1930 & 1279 & 1933 & $\begin{array}{c}\text { Benzeneacetaldehyde, alpha.-ethylidene- } \\
\text { Ketones }\end{array}$ & & 0.045 & MS, RI \\
\hline 707 & 1303 & 708 & 1301 & 2-Butanone, 3-hydroxy- & 0.329 & 0.361 & MS, RI \\
\hline 986 & & 986 & & 3-Octanone & 0.055 & & MS, RI \\
\hline 1027 & & 1043 & & 1,2-Cyclopentanedione, 3-methyl- & & 0.221 & MS \\
\hline 1067 & 1958 & 1067 & 1957 & Ethanone, 1-(1H-pyrrol-2-yl)- & & 0.233 & MS,RI \\
\hline 1071 & & 1071.6 & & Acetophenone & & 0.051 & MS,RI \\
\hline \multirow[t]{4}{*}{1114} & & 1117 & & 1,7-Octadien-3-one, 2-methyl-6-methylene- & 0.037 & & MS,RI \\
\hline & 1448 & & & $\begin{array}{c}\text { Cyclohexanone, 5-methyl-2-(1-methylethyl)-, } \\
\text { (2S-trans)- }\end{array}$ & 0.037 & & MS \\
\hline & 1486 & & 1489 & Ethanone, 1-(2-furanyl)- & & 0.119 & MS, RI \\
\hline & 1487 & & & 1-(3H-Imidazol-4-yl)-ethanone & & 0.226 & MS \\
\hline \multirow[t]{5}{*}{1151} & & 1151 & & $\begin{array}{l}\text { 4H-Pyran-4-one, 2,3-dihydro-3,5-dihydroxy-6- } \\
\text { methyl- }\end{array}$ & & 0.107 & MS, RI \\
\hline & 1569 & & 1573 & 2-Cyclopentene-1,4-dione & & 1.536 & MS, RI \\
\hline & 1588 & & 1587 & Ethanone, 1-(2-pyridinyl)- & & 0.526 & MS, RI \\
\hline & 1814 & & & 1,2-Cyclopentanedione, 3-methyl- & & 0.134 & MS \\
\hline & 2012 & & & $\begin{array}{l}\text { 2(3H)-Furanone, dihydro-3-hydroxy-4,4- } \\
\text { dimethyl- }\end{array}$ & & 0.101 & MS \\
\hline
\end{tabular}


Table 5. Cont.

\begin{tabular}{|c|c|c|c|c|c|c|c|}
\hline \multicolumn{2}{|c|}{$\begin{array}{c}\text { RI } \\
\text { Calculated a }^{\text {a }} \\
\end{array}$} & \multicolumn{2}{|c|}{$\begin{array}{c}\text { RI } \\
\text { Literature }^{\mathbf{b}} \\
\end{array}$} & \multirow[t]{2}{*}{ Compound } & \multirow{2}{*}{$\begin{array}{c}\operatorname{Raw}^{\mathrm{c}} \\
\left(\mathrm{ppm}^{\mathrm{d}}\right)\end{array}$} & \multirow{2}{*}{$\frac{\text { Toasted }^{\mathrm{c}}}{\left(\mathrm{ppm}^{\mathrm{d}}\right)}$} & \multirow[t]{2}{*}{ ID $^{\mathrm{e}}$} \\
\hline DB5 & WAX & DB5 & WAX & & & & \\
\hline \multicolumn{8}{|c|}{ Carboxylic acids } \\
\hline \multirow[t]{6}{*}{613} & 1415 & 610 & 1415 & Acetic acid & 2.163 & 5.939 & MS, RI \\
\hline & 1608 & & & Benzoic acid, hydrazide & 0.040 & & MS \\
\hline & 1616 & & & Butanoic acid, 4-hydroxy- & 0.184 & 0.656 & MS \\
\hline & 1829 & & 1827 & Hexanoic acid & 0.282 & 0.089 & MS, RI \\
\hline & 2028 & & 2030 & Octanoic Acid & 0.110 & 0.209 & MS, RI \\
\hline & & & & Esters & & & \\
\hline 719 & 972 & 720 & 971 & Butanoic acid, methyl ester & 0.019 & & MS, RI \\
\hline 774 & 1007 & 776 & 1007 & Acetic acid, 2-methylpropyl ester & 0.191 & 0.096 & MS, RI \\
\hline 826 & 1076 & 825 & 1075 & Pentanoic acid, methyl ester & 0.166 & & MS, RI \\
\hline 878 & 1115 & 877 & 1115 & 1-Butanol, 3-methyl-, acetate & 0.282 & 0.404 & MS, RI \\
\hline 880 & & 880 & & 1-Butanol, 2-methyl-, acetate & & 0.260 & MS, RI \\
\hline 912 & 1616 & 912 & 1617 & Butyrolactone & 0.297 & & MS, RI \\
\hline \multirow[t]{2}{*}{925} & & 926 & & Hexanoic acid, methyl ester & 0.120 & & MS, RI \\
\hline & 1320 & & 1317 & Heptanoic acid, ethyl ester & 0.061 & & MS, RI \\
\hline 1045 & & & & Pantolactone & & 0.192 & MS \\
\hline 1099 & 1607 & 1100 & 1605 & Benzoic acid, methyl ester & 0.162 & & $\mathrm{MS}, \mathrm{RI}$ \\
\hline \multirow[t]{2}{*}{1167} & & 1165 & & Acetic acid, phenylmethyl ester & & 0.096 & $\mathrm{MS}, \mathrm{RI}$ \\
\hline & & & & Alcohols & & & \\
\hline \multirow[t]{2}{*}{615} & 1085 & 618 & 1086 & 1-Propanol, 2-methyl- & 0.389 & 0.229 & MS, RI \\
\hline & 918 & & 920 & Isopropyl Alcohol & 0.080 & & MS, RI \\
\hline 734 & 1189 & 730 & 1190 & 1-Butanol, 3-methyl- & 0.173 & & MS, RI \\
\hline 738 & 1189 & & & 1-Butanol, 2-methyl-, (+/-)- & 0.381 & & MS \\
\hline 767 & 1231 & 766 & 1232 & 1-Pentanol & 0.595 & 0.536 & MS, RI \\
\hline \multirow[t]{3}{*}{797} & 1584 & 800 & 1583 & 2,3-Butanediol $\left[R-\left(R^{*}, R^{*}\right)\right]$ - & 17.039 & 17.448 & MS, RI \\
\hline & 1088 & & & 1,2-Cyclopentanediol, trans- & & 0.030 & MS \\
\hline & 1136 & & 1136 & 1-Butanol & 0.050 & 0.062 & MS, RI \\
\hline 858 & 1635 & 858 & 1638 & 2-Furanmethanol & & 0.747 & MS, RI \\
\hline \multirow[t]{2}{*}{955} & 1700 & 953 & & 2-Furanmethanol, 5-methyl- & & 0.096 & MS, RI \\
\hline & 1334 & & 1335 & 1-Hexanol & 0.201 & & MS, RI \\
\hline 1039 & 1866 & 1039 & 1866 & Benzyl alcohol & 0.332 & 1.019 & MS, RI \\
\hline \multirow[t]{5}{*}{1051} & & & & 3-Octen-1-ol & 0.069 & & MS \\
\hline & 1379 & & 1379 & Ethanol, 2-butoxy- & 0.063 & & MS, RI \\
\hline & 1580 & & & $\begin{array}{c}\text { Cyclohexanol, 5-methyl-2-(1-methylethyl)-, } \\
(1 \alpha, 2 \beta, 5 \alpha)-\end{array}$ & & 0.743 & MS \\
\hline & 1885 & & & $3,6,9,12$-Tetraoxatetradecan-1-ol & 0.008 & & MS \\
\hline & 1903 & & 1905 & Phenylethyl Alcohol & 0.065 & 0.166 & MS, RI \\
\hline 1684 & & & & $\begin{array}{c}\text { 2,6-Bis(1,1-dimethylethyl)-4- } \\
\text { (1-oxopropyl)phenol }\end{array}$ & 0.010 & 0.014 & MS \\
\hline
\end{tabular}


Table 5. Cont.

\begin{tabular}{|c|c|c|c|c|c|c|c|}
\hline \multicolumn{2}{|c|}{$\begin{array}{c}\text { RI } \\
\text { Calculated }^{\mathrm{a}} \\
\end{array}$} & \multicolumn{2}{|c|}{$\begin{array}{c}\text { RI } \\
\text { Literature }^{b}\end{array}$} & \multirow[t]{2}{*}{ Compound } & \multirow{2}{*}{$\frac{\operatorname{Raw}^{\mathrm{c}}}{\left(\mathrm{ppm}^{\mathrm{d}}\right)}$} & \multirow{2}{*}{$\begin{array}{c}\text { Toasted }^{\mathrm{c}} \\
\left(\mathrm{ppm}^{\mathrm{d}}\right)\end{array}$} & \multirow[t]{2}{*}{ ID $^{\mathrm{e}}$} \\
\hline DB5 & WAX & DB5 & WAX & & & & \\
\hline \multicolumn{8}{|c|}{ Hydrocarbons } \\
\hline 647 & 923 & 648 & 924 & Benzene & 0.043 & 0.086 & MS, RI \\
\hline \multirow[t]{3}{*}{765} & 1029 & 765 & 1029 & Toluene & 0.052 & 0.110 & MS, RI \\
\hline & 1094 & & & Undecane & 0.030 & & MS \\
\hline & 1126 & & 1123 & Benzene, 1,3-dimethyl- & 0.050 & & MS, RI \\
\hline 862 & & 863 & & Ethylbenzene & 0.022 & & MS, RI \\
\hline \multirow[t]{2}{*}{872} & & & & Cyclopropane, propyl- & & 0.324 & MS \\
\hline & 1166 & & 1164 & $p$-Xylene & 0.010 & & MS, RI \\
\hline 893 & 1237 & 894 & 1236 & Styrene & 0.108 & 0.084 & MS, RI \\
\hline 899 & & & & Nonane & 0.020 & 0.074 & MS \\
\hline \multirow[t]{2}{*}{931} & & & & 2,4-Octadiene & & 0.057 & MS \\
\hline & 1238 & & 1264 & 1,3,5,7-Cyclooctatetraene & & 0.300 & MS \\
\hline 996 & & 996 & & Benzene, 1,3,5-trimethyl- & 0.040 & & MS, RI \\
\hline 999 & 997 & & & Decane & 0.096 & 0.089 & MS \\
\hline \multirow[t]{3}{*}{1033} & 1079 & 1030 & & D-Limonene & 0.025 & 0.048 & MS, RI \\
\hline & 1412 & & & Benzene, 2-ethyl-1,4-dimethyl- & 0.011 & & MS \\
\hline & 1413 & & & Benzene, 1-ethyl-2,3-dimethyl- & 0.027 & & MS \\
\hline 1125 & & 1120 & & Benzene, 1,2,3,4-tetramethyl- & 0.040 & & MS, RI \\
\hline 1303 & & 1300 & & Tridecane & 0.015 & 0.027 & MS, RI \\
\hline \multirow[t]{3}{*}{1399} & & 1400 & & Tetradecane & 0.015 & 0.040 & MS, RI \\
\hline & 2056 & & & 1,4,7,10,13,16-Hexaoxacyclooctadecane & 0.029 & & MS \\
\hline & & & & Pyrazines & & & \\
\hline 826 & 1247 & 827 & 1247 & Pyrazine, methyl- & & 3.745 & MS, RI \\
\hline 896 & 1487 & & 1491 & 2,3,5-Trimethyl-6-ethylpyrazine & & 0.221 & MS, RI \\
\hline \multirow[t]{2}{*}{915} & 1306 & 915 & 1308 & Pyrazine, 2,5-dimethyl- & & 7.344 & MS, RI \\
\hline & 1312 & & 1314 & Pyrazine, 2,6-dimethyl- & & 1.297 & MS, RI \\
\hline 998 & 1365 & 998 & 1367 & Pyrazine, 2-ethyl-6-methyl- & & 0.701 & MS, RI \\
\hline \multirow[t]{3}{*}{1002} & 1384 & 1005 & 1381 & Pyrazine, trimethyl- & & 1.685 & MS, RI \\
\hline & 1330 & & 1326 & Pyrazine, 2,3-dimethyl- & & 0.287 & MS, RI \\
\hline & 1371 & & 1367 & Pyrazine, 2-ethyl-5-methyl- & & 1.494 & MS, RI \\
\hline 1078 & 1425 & 1078 & 1430 & Pyrazine, 3-ethyl-2,5-dimethyl- & & 1.907 & MS, RI \\
\hline 1085 & & 1085 & & Pyrazine, 2-ethyl-3,5-dimethyl- & & 0.143 & MS, RI \\
\hline \multirow[t]{2}{*}{1086} & 1458 & 1088 & 1457 & Pyrazine, tetramethyl- & 0.106 & 0.597 & MS, RI \\
\hline & 1411 & & 1415 & Pyrazine, 2,6-diethyl- & & 0.066 & MS, RI \\
\hline \multirow[t]{2}{*}{1094} & & 1091 & & Pyrazine, 2,5-diethyl- & & 0.106 & MS, RI \\
\hline & 1472 & & 1485 & Pyrazine, 2-ethenyl-6-methyl- & & 0.105 & MS, RI \\
\hline 1147 & 1609 & & 1605 & 5H-5-Methyl-6,7-dihydrocyclopentapyrazine & & 0.500 & MS, RI \\
\hline 1153 & 1475 & 1156 & 1478 & Pyrazine, 2,3-diethyl-5-methyl- & & 0.130 & MS, RI \\
\hline 1157 & & 1156 & & Pyrazine, 3,5-diethyl-2-methyl- & & 0.245 & MS, RI \\
\hline 1161 & & 1357 & & 2,5-Dimethyl-3-n-pentylpyrazine & & 0.021 & MS \\
\hline \multirow{3}{*}{1318} & 1683 & & 1679 & 1-(6-Methyl-2-pyrazinyl)-1-ethanone & & 0.146 & MS, RI \\
\hline & & 1254 & & Pyrazine, 2-butyl-3,5-dimethyl- & & 0.019 & MS \\
\hline & 1705 & & 1740 & Pyrazinamide & & 0.208 & MS \\
\hline
\end{tabular}


Table 5. Cont.

\begin{tabular}{|c|c|c|c|c|c|c|c|}
\hline \multicolumn{2}{|c|}{$\begin{array}{c}\text { RI } \\
\text { Calculated }^{\mathrm{a}}\end{array}$} & \multicolumn{2}{|c|}{$\begin{array}{c}\text { RI } \\
\text { Literature }^{\mathrm{b}}\end{array}$} & \multirow[t]{2}{*}{ Compound } & \multirow{2}{*}{$\begin{array}{l}\operatorname{Raw}^{c} \\
\left(\operatorname{ppm}^{\mathrm{d}}\right)\end{array}$} & \multirow{2}{*}{$\begin{array}{c}\text { Toasted }^{\mathrm{c}} \\
\left(\text { ppm }^{\mathrm{d}}\right) \\
\end{array}$} & \multirow[t]{2}{*}{ ID $^{\mathrm{e}}$} \\
\hline DB5 & WAX & DB5 & WAX & & & & \\
\hline \multicolumn{8}{|c|}{ Pyrroles and Furans } \\
\hline 811 & 1165 & 811 & 1168 & 1H-Pyrrole, 1-ethyl- & & 0.144 & MS, RI \\
\hline 847 & & 841 & & 1H-Pyrrole, 3-methyl- & & 0.082 & MS, RI \\
\hline 948 & & & & 1H-Pyrrole, 1-butyl- & & 0.021 & MS \\
\hline 891 & 1122 & 892.1 & 1123 & 2-n-Butylfuran & & 0.707 & MS, RI \\
\hline \multirow[t]{2}{*}{991} & 1211 & 991 & 1213 & Furan, 2-pentyl- & 0.077 & 0.236 & MS, RI \\
\hline & 1037 & & & 2,3-Dihydrofuran & & 0.008 & MS \\
\hline 1016 & 2004 & 1016 & 2006 & 1H-Pyrrole-2-carboxaldehyde & & 0.114 & MS, RI \\
\hline \multirow[t]{4}{*}{1076} & 2020 & 1076 & 2020 & 2-Pyrrolidinone & & 0.477 & MS, RI \\
\hline & 1675 & & 1678 & 2-Pyrrolidinone, 1-methyl- & & 0.057 & MS, RI \\
\hline & & & & Miscellaneous & & & \\
\hline & 632 & & 643 & Methanethiol & & 0.046 & MS \\
\hline 559 & 701 & & & Dimethyl sulfide & 0.052 & 0.071 & MS \\
\hline 567 & & 531 & & Methylene chloride & 0.844 & 0.474 & MS \\
\hline 612 & 1009 & 615 & 1010 & Trichloromethane & 0.059 & 0.028 & MS, RI \\
\hline 724 & & & & Propanamide, $N, N$-dimethyl- & 0.025 & & MS \\
\hline 850 & & 850 & & Oxazole, trimethyl- & & 0.034 & MS, RI \\
\hline \multirow[t]{4}{*}{1020} & & 1020 & & Benzene, 1,4-dichloro- & 0.450 & 0.253 & MS, RI \\
\hline & 1419 & & & Benzene, 1,2-dichloro- & & 0.450 & MS \\
\hline & 1710 & & & 6-Aminoindoline & & 0.136 & MS \\
\hline & 1923 & & 1912 & Benzonitrile, 2-methyl- & & 0.060 & MS \\
\hline \multirow[t]{2}{*}{1537} & & & & $\begin{array}{l}N \text {-(2-Benzoyl-4-nitrophenyl)-4-tert- } \\
\text { butylbenzamide }\end{array}$ & & 0.041 & MS \\
\hline & 2051 & & & Octaethylene glycol & 0.007 & & MS \\
\hline
\end{tabular}

Notes: ${ }^{\mathrm{a}}$ Calculated Kovats retention index on DB- 5 or Wax column using a n-alkanes series; ${ }^{\mathrm{b}}$ Reported Kovats retention index on library NIST; ${ }^{c}$ Average of 3 replicates. Coefficient of variation $<9.2 \%$; ${ }^{\mathrm{d}}$ Semi-quantitative values. ${ }^{\mathrm{e}} \mathrm{MS}=$ tentative identification by comparison to mass spectra in NIST library, RI = identification by comparison of calculated Kovats retention index to that reported in literature.

The most abundant alcohol identified in P. serotina seeds was 2,3-butanediol, which was also the major volatile compound in raw (17.03 ppm) and toasted seeds (17.44 ppm). This alcohol is the major volatile component of sweet kernels and has been associated to their characteristic taste [34]. 2,3-butanediol is also found in cheeses [45] and honey [46]. Recently, it has been reported that this metabolite is abundant in roasted Trichosanthes kirilowii seeds, however it was absent in the raw seeds, which suggests that 2,3-butanediol was generated during the toasting process [47]. In contrast, our results indicated that 2,3-butanediol levels in black cherry seeds were not significantly different before and after toasting. Benzyl alcohol, predominantly found in toasted seeds, imparts a sharp, burning taste and a faint aroma in toasted almonds [48]. This compound was previously detected as one of the major volatiles of black cherry leaves and also displays a vasodilator effect [8]. Regarding 1-pentanol, detected in both raw and toasted seeds, it has been proposed that it derives from the oxidation of linoleic acid, and its concentration increases with storage time in almonds [49]. 
Acetic acid was the main volatile carboxylic acid found in raw and toasted $P$ serotina seeds. This carboxylic acid can be regarded as a result of long-chain fatty acids degradation and it is often related to a strong sour odor [50]. Esters were detected in lesser quantities, some of them, such as methyl esters of short chain fatty acids, were found in raw seeds, but not in toasted seeds. Hydrocarbons and ketones were minor compounds in both raw and toasted seeds.

Pyrazines and pyrroles were other major volatiles identified exclusively in toasted black cherry seeds. These compounds are produced during the toasting process from free amino acids and monosaccharides by the Maillard reaction through Strecker degradation [33], particularly, ethyl-methyl-, ethyldimethyl-, and diethylmethylpyrazines, are formed in the reaction of glucose and fructose with alanine and glycine [51]. The most abundant pyrazine in black cherry toasted seeds was 2,5-dimethylpyrazine, followed by methylpyrazine, 3-ethyl-2,5-dimethylpyrazine and trimethylpyrazine. The first one has been previously reported as a major pyrazine in roasted hazelnuts [33] and toasted almonds [48]. Pyrazines contribute to the characteristic toasted aroma in chesnuts [52], and peanuts [53], almonds [54], and pyrroles significantly are responsible for the characteristic toasted aroma of different thermal treated foods [44].

\section{Experimental Section}

Reagents and standards were purchased from Sigma-Aldrich (St. Louis, MO, USA), unless otherwise noted. Solvents were purchased from Baker-Mallincrodt (JT Baker, Mallinckrodt Baker Inc., Phillipsburg, NJ, USA).

\subsection{Samples}

Ripe black cherry fruits were cultivated in Huejotzingo, Puebla (México) and harvested in May of 2011. Subsequently, the seeds were removed from the pulp with plastic knives, washed and allowed to dry at $25^{\circ} \mathrm{C}$ during $48 \mathrm{~h}$. Finally, the seeds were stored at $-70{ }^{\circ} \mathrm{C}$ until analysis.

All the analyses were carried out on raw and toasted seeds. Toasting was performed in a comal (traditional Mexican iron griddle) during $20 \mathrm{~min}$ at $125^{\circ} \mathrm{C}$. Then, the seeds were rotated frequently to avoid overheating and to obtain homogenous toasting. Raw and toasted seeds were cracked and opened with pliers to get the kernel.

Taking into account that black cherry toasted seeds are consumed by Mexican people as snacks, almonds (Prunus dulcis) and peanuts (Arachis hypogaea) samples were analyzed for comparative purposes. Both seeds were purchased in a local market in the city of Queretaro, Queretaro (México).

\subsection{Chemical Proximate Analysis}

Chemical proximate analysis was carried out on raw and toasted black cherry seeds, almonds and peanuts by using AOAC methods (AOAC, 2000). Moisture, protein, fat, ash, crude fiber and carbohydrates contents were determined by methods 950.46, 928.08, 960.39, 920.153 and 985.29, respectively. Total carbohydrate content (on dry weight basis) was calculated by difference $[100-($ protein + lipids + ash + crude fiber $)]$. 


\subsection{Determination of Vitamins $A, C$, and $E$}

Vitamins and carotenes were determined by using AOAC methods (2000) for vitamin A (960.46), vitamin C (967.22), and vitamin E (992.03).

\subsection{Mineral Content}

Sodium, potassium, calcium, magnesium and iron content of samples was determined by method 985.35 described in Association of Official Analytical Chemists (AOAC, 2000). Phosphorous was determined according to method 965.17 (AOAC, 2000).

\subsection{In Vitro Protein Digestibility}

The in vitro protein digestibility of dry samples was estimated by using the methodology of Hsu et al. [55] and applying the equation $Y=234.84-22.56 X$, where $Y$ is the in vitro protein digestibility $(\%)$ and $X$ is the $\mathrm{pH}$ of the protein sample suspension, after proteolysis with a multienzyme system consisting of porcine pancreatic trypsin type IX, bovine pancreatic chymotrypsin type II, and porcine intestinal peptidase grade III. The average value of three replicates is reported.

\subsection{Amino Acid Analysis}

Proteins were hydrolyzed in hydrochloric acid (JT Baker) and amino acids were analyzed using a HPLC autoanalyzer (Waters 2487, Millipore, MA, USA), according to Bidlingmeyer et al. [56].

\subsection{Nutritional Quality of Proteins}

For predicting dietary protein quality, the in vitro protein digestibility and amino acid composition were used to calculate PDCAAS (protein digestibility corrected amino acid score) according to the FAO/WHO [30] prescribed formula:

$$
\text { PDCAAS }=\text { Amino acid score }(\text { of the most limiting AA }) \times \text { Digestibility }
$$

\subsection{Volatile Compounds Analysis}

Extraction conditions were based on previously published work of Krist et al. [52] and Agila et al. [54] with some modifications. Fresh seeds (15 g) were blended in an electric blender (model O-20, Osterizer $^{\mathrm{TM}}$, Boca Raton, FL, USA) for $15 \mathrm{~s}$. The ground seeds $(1 \mathrm{~g})$ were transferred into a $20 \mathrm{~mL}$ vial, along with $7 \mu \mathrm{g}$ of a menthol solution $(0.01 \% \mathrm{w} / \mathrm{w})$ as an internal standard and sealed with a Nickel-aluminum crimp cap provided with a needle-pierceable polytetrafluroethylene/silicone septum. Vials were pre-equilibrated for $60 \mathrm{~min}$ at $50{ }^{\circ} \mathrm{C}$. Solid-phase microextraction (SPME) was performed with a $75 \mathrm{~mm}$ divinylbenzene/carboxen/polydimethylsiloxane (DVB/CAR/PDMS) fiber (Supelco Co, Bellefonte, PA, USA). Extractions and injections were performed using a MPS2 autosampler (Gerstel, Linthicum-Baltimore City, MD, USA) fitted with a vial heater. The fiber was exposed for $10 \mathrm{~min}$ in the head space of the vial for analytes adsorption. Subsequently, the fiber was removed from the vial and placed into the chromatograph injection port for desorption. 
Gas chromatography separation and quantification was carried out using an Agilent GC 7890A series intrumenbt (Agilent Technologies, Inc., Santa Clara, CA, USA) equipped with a flame ionization detector (GC-FID). Injection port and detector temperature was $230{ }^{\circ} \mathrm{C}$. The injector was operated in the splitless mode. The capillary columns used were an HP-5 $60 \mathrm{~m} \times 0.32 \mathrm{~mm}$ i.d., $0.32 \mu \mathrm{m}$ film thickness; Agilent Technologies, Inc.) and a DB-Wax (60 m $\times 0.32 \mathrm{~mm}$ i.d., $0.32 \mu \mathrm{m}$ film thickness; Agilent Technologies, Inc.). Oven temperature was programmed at initial temperature of $40{ }^{\circ} \mathrm{C}$ for 5 min, then raised at $5{ }^{\circ} \mathrm{C} / \mathrm{min}$ to $230^{\circ} \mathrm{C}$ and hold for $15 \mathrm{~min}$. Helium was used as the carrier gas at a constant flow rate of $1 \mathrm{~mL} / \mathrm{min}$. A semi-quantitative evaluation was achieved by comparing individual peak area from GC-FID response to that of the internal standard. Each tabulated value corresponds to the average of 3 extraction replicates.

Gas Chromatography Mass Spectrometry (GC/MS) analysis was carried out using an Agilent GC 7890A series equipped with an Agilent 5975C Mass Spectrometer in electron impact mode (EI) and a quadrupole analyzer. The temperatures of ion source and quadrupole were 230 and $250{ }^{\circ} \mathrm{C}$ respectively. Transfer line was set at $280{ }^{\circ} \mathrm{C}$. Full scan mode was used in a range of 33-300 uma, at a scan rate of $5.2 / \mathrm{s}$, with an ionization voltage of $70 \mathrm{eV}$. The same chromatographic conditions as for GC-FID were used. MSD ChemStation E.01.00.237 software (Agilent Technologies, Inc.) was employed for data analysis.

Identification of volatile compounds was performed by comparing their mass spectra with those in the NIST Mass Spectral Library (National Institute of Standards and Technology, Gaithersburg, MD, USA). Retention indices of all the volatile compounds were determined by the modified Kovats method reported by Van den Dool and Kratz [57]. MS identification was confirmed by comparing Kovats retention indices (RI) to RI reported in the literature [58].

\subsection{Statistical Analysis}

Results of the experiments are expressed as the mean \pm standard deviation (SD) from $n=3$ experiments. The data were analyzed by a one-way ANOVA and the Tukey test. Differences between the means were considered to be significant when $p<0.05$. Statistical treatment of data was performed with the program Prism 4.0 (GraphPad Software, San Diego, CA, USA).

\section{Conclusions}

This study demonstrates that black cherry seeds have a significant content of minerals, particularly potassium, lipids and proteins. Their PDCAAS values suggest that they might be considered as a complementary source of protein. Additionally, the gas chromatography/mass spectrometry analysis allowed identification of 59 and 99 volatile compounds in the raw and toasted seeds, respectively. The major volatile components identified in raw and toasted P. serotina seeds were 2,3-butanediol and benzaldehyde. The results derived from this study indicate that these seeds have nutraceutical properties attributed to their high protein and potassium content and the presence of bioactive compounds such as benzaldehyde. 


\section{Acknowledgments}

Leticia García-Aguilar acknowledges Consejo Nacional de Ciencia y Tecnología (CONACYT) for her Ph. D. scholarship. This work was supported by grants (a) FOFI-UAQ-2012 (Project FCQ-2012-25) and (b) INFR-2014-01-226186 from CONACYT assigned to Alejandra Rojas Molina.

\section{Author Contributions}

Leticia García-Aguilar extracted and analyzed the volatile compounds and assessed nutritional protein quality as part of her doctoral degree thesis. Alejandra Rojas-Molina designed this project, coordinated all the activities, and supervised the chemical studies. César Ibarra-Alvarado helped in the design of experiments. Pedro A. Vázquez-Landaverde conducted the final identification of volatile compounds. Juana I. Rojas-Molina supervised the nutrimental analysis. Francisco J. Luna-Vázquez was in charge of the fruit collection and identification and revised the manuscript. Miguel A. Zavala-Sánchez contributed with the preparation of manuscript.

\section{Conflicts of Interest}

The authors declare no conflict of interest.

\section{References}

1. McVaugh, R. A revision of the North American black cherries (Prunus serotina Ehrh) and relatives. Brittonia 1951, 7, 279-315.

2. Ordaz-Galindo, A.; Wesche-Ebeling, P.; Wrolstad, R.E.; Rodríguez- Saona, L.; Argaiz-Jamet, A. Purification and identification of Capulin (Prunus serotina Ehrh) anthocyanins. Food Chem. 1999, 65, 201-206.

3. Starfinger, U.; Kowarik, I.; Rode, M.; Schepker, H. From desirable ornamental plant to pest to accepted addition to the flora?-The perception of an alien tree species through the centuries. Biol. Invasions 2003, 5, 323-335.

4. Vázquez-Yanes, C.; Batis-Muñoz, A.I.; Alcocer-Silva, M.I.; Gual-Díaz M.; Sánchez-Dirzo, C. Árboles y arbustos nativos potencialmente valiosos para la restauración ecológica y la reforestación. PROYECTO J-084-CONABIO. Comisión Nacional para el Conocimiento y Uso de la Biodiversidad (CONABIO). Available online: http:/www.conabio.gob.mx/conocimiento/ info_especies/arboles/doctos/inicio.pdf (accessed on 15 November 2014).

5. Argueta, A. Monografías de plantas medicinales. In Atlas de las Plantas de la Medicina Tradicional Mexicana; Instituto Nacional Indigenista: México, México, 1994; Volume 1, pp. 319-320.

6. Martínez, M. Plantas cuyas propiedades están comprobadas científicamente. In Plantas Medicinales de México; Editorial Botas: México, México, 1991; Volume 1, pp. 61-62.

7. McCune, L.M.; Johns, T. Antioxidant activity relates to plant part, life form and growing condition in some diabetes remedies. J. Ethnopharmacol. 2007, 112, 461-469.

8. Ibarra-Alvarado, C.; Rojas, A.; Luna, F.; Rojas, J.I.; Rivero-Cruz, B.; Rivero-Cruz, F. Vasorelaxant constituents of the leaves of Prunus serotina "capulín". Rev. Latinoam. Quim. 2009, 37, 164-173. 
9. Luna-Vázquez, F.J.; Ibarra-Alvarado, C.; Rojas-Molina, A.; Rojas-Molina, J.I.; Yahia, E.M.; Rivera-Pastrana, D.M.; Rojas-Molina, A.; Zavala-Sánchez, M.A. Nutraceutical value of black cherry Prunus serotina Ehrh. Fruits: Antioxidant and antihypertensive properties. Molecules 2013, 18, 14597-14612.

10. Alveano-Aguerrebere, I.; Rojas-Molina, A.; Oomah, D.V.; Drover, J.C.G. Characteristics of Prunus serotina seed oil. Food Chem. 2011, 124, 983-990.

11. Calder, P.C.; Grimble, R.F. Polyunsaturated fatty acids, inflammation and immunity. Eur. J. Clin. Nutr. 2002, 56, S14-S19.

12. Kinsella, E.J.; Lokesh, B.; Stone R.A. Dietary n-3 polyunsaturated fatty acids and amelioration of cardiovascular disease: Possible mechanisms1. Am. J. Clin. Nutr. 1990, 52, 1-28.

13. Moon, H.S.; Guo, D.D.; Lee, H.G.; Choi, Y.J.; Kang, J.S.; Jo, K.; Eom, J.M.; Yun, C.H. Alpha-eleostearic acid suppresses proliferation of MCF-7 breast cancer cells via activation of PPAR $\gamma$ and inhibition of ERK 1/2. Cancer Sci. 2010, 101, 396-402.

14. Swain, E.; Poulton. J.E. Utilization of amygdalin during seedling development of Prunus serotina. Plant Physiol. 1994, 106, 437-445.

15. Khattab R.Y.; Arntfield, S.D. Nutritional quality of legume seeds as affected by some physical treatments 2. Antinutritional factors. LWT-Food Sci. Technol. 2009, 42, 1113-1118.

16. Cao, J.; Qi, M.; Zhang, Y.; Zhou, Z.; Shao, Q.; Fu, R. Analysis of volatile compounds in Curcuma wenyujin Y.H. Chen et C. Ling by headspace solvent microextraction-gas chromatography-mass spectrometry. Anal. Chim. Acta 2006, 561, 88-95.

17. Ahrens, S.; Venkatachalam, M.; Mistry, A.M.; Lapsley, K.; Sathe, S.K. Almond (Prunus dulcis L.) protein quality. Plant Food Hum. Nutr. 2005, 60, 123-128.

18. Singh, B.; Singh, U. Peanut as a source of protein for human foods. Plant Food Hum. Nutr. 1991, $41,165-177$.

19. Kamel, B.S.; Kakuda, Y. Characterization of the seed oil and meal from apricot, cherry, nectarine, peach and plum. J. Am. Oil Chem. Soc. 1992, 69, 492-424.

20. Venkatachalam, M.; Sathe, S.K. Chemical composition of selected edible nut seeds. J. Agric. Food Chem. 2006, 54, 4705-4714.

21. Ros, E.; Mataix, J. Fatty acid composition of nuts-Implications for cardiovascular health. Br. J. Nutr. 2006, 96, S29-S35.

22. Caglarirmak, N.; Batkan, A.C. Nutrients and biochemistry of nuts in different consumption types in Turkey. J. Food Process. Pres. 2005, 29, 407-423.

23. Lazos, E.S. Nutritional, fatty acid, and oil characteristics of pumpkin and melon seeds. J. Food Sci. 1986, 51, 1382-1383.

24. Anderson, J.W.; Smith, B.M.; Gustafson, N.J. Health benefits and practical aspects of high-fiber diets. Am. J. Clin. Nutr. 1994, 59, 1242S-1247S.

25. Chandalia, M.; Abhimanyu, G.; Lutjohann, D.; Bergmann, V.K.; Scott, M.G.; Brinkley, L.J. Beneficial effects of high dietary fiber intake in patients with type 2 diabetes mellitus. N. Engl. J. Med. 2000, 342, 1392-1398.

26. Chitra, U.; Singh, U.; Venkateswara, P.R. Phytic acid, in vitro protein digestibility, dietary fibers and minerals of pulses as influenced by processing methods. Plant Food Hum. Nutr. 1996, 49, 307-316. 
27. Gamel, T.H.; Linssen, J.P.; Mesallam, A.S.; Damir, A.A.; Shekib, L.A. Effect of seed treatments on the chemical composition of two amaranth species: Oil, sugars, fibres, minerals and vitamins. J. Sci. Food Agric. 2006, 86, 82-89.

28. Kornsteiner, M.; Wagner, K.H.; Elmadfa, I. Tocopherols and total phenolics in 10 different nut types. Food Chem. 2006, 98, 381-387.

29. Muñoz Chávez, M. Oleaginosas. In Tablas de valor nutritivo de los alimentos de mayor consumo en Latinoamérica; Pax: México, D.F., México, 1996; p. 44.

30. FAO/WHO. Findings and recommendations of the 2011 FAO expert consultation on protein quality Evaluation in human nutrition. In Dietary Protein Quality Evaluation in Human Nutrition, Report of an FAO Expert Consultation; Food and Agricultural Organization of the United Nations: Rome, Italy, 2013; Volume 92, p. 19.

31. Hughes, G.J.; Ryan, D.J.; Mukherjea, R.; Schasteen, C.S. Protein digestibility-corrected amino acid scores (PDCAAS) for soy protein isolates and concentrate: Criteria for evaluation. J. Agric. Food Chem. 2011, 59, 12707-12712.

32. Fernandes, D.C.; Freitas, J.B.; Czedera, L.P.; Naves, M.M.V. Nutritional composition and protein value of the baru (Dipteryx alata Vog.) almond from the Brazilian Savanna. J. Sci. Food Agric. 2010, 90, 1650-1655.

33. Alasalvar, C.; Shahidi, F.; Cadwallader, K.R. Comparison of natural and roasted Turkish Tombul hazelnut (Corylus avellana L.) volatiles and flavor by DHA/GC/MS and descriptive sensory analysis. J. Agric. Food Chem. 2003, 51, 5067-5072.

34. Wirthensohn, M.G.; Chin, W.L.; Franks, T.K.; Baldock, G.; Ford, C.M.; Sedgley, M. Investigation of flavour compounds from sweet, semi-bitter and bitter almond kernels. Options Méditerranéennes 2010, 94, 117-122.

35. Chandra, A.; Nair, M.G. Quantification of benzaldehyde and its precursors in Montmorency cherry (Prunus cerasus L.) kernels. Phytochem. Anal. 1993, 4, 120-123.

36. Santamour, F.S. Amygdalin in Prunus leaves. Phytochemistry 1998, 47, 1537-1538.

37. Swain, E.; Li, C.P.; Poulton, J.E. Development of the potential for cyanogenesis in maturing black cherry (Prunus serotina Ehrh.) fruits. Plant. Physiol. 1992, 98, 1423-1428.

38. Poulton, J.E. Enzymology of cyanogenesis in rosaceous stone fruits. In $\beta$-Glucosidases: Biochemistry and Molecular Biology, ACS Symposium Series No. 533; Esen, A., Ed.; American Chemical Society: Washington, DC, USA, 1993; pp. 170-190.

39. Sánchez-Pérez, R.; Sáenz Belmonte, F.; Borch, J.; Dicenta, F.; Møller, B.L.; Jørgensen, K. Prunasin hydrolases during fruit development in sweet and bitter almonds. Plant Physiol. 2012, 158, 1916-1932.

40. Swain, E.; Li, C.P.; Poulton, J.E. Tissue and subcellular localization of enzymes catabolizing (R)-Amygdalin in mature Prunus serotina seeds. Plant Physiol. 1992, 100, 291-300.

41. Adamiec, J.; Rössner, J.; Cejpek, J.V.K.; Šavel, J. Minor strecker degradation products of phenylalanine and phenylglycine. Eur. Food Res. Technol. 2001, 212, 135-140.

42. Chu, F.L.; Yaylayan, V.A. Model studies on the oxygen-induced formation of benzaldehyde from phenylacetaldehyde using pyrolysis GC-MS and FTIR. J. Agric. Food Chem. 2008, 56, 10697-10704.

43. Siegmund, B.; Murkovic, M. Changes in chemical composition of pumpkin seeds during the roasting process for production of pumpkin seed oil (Part 2: volatile compounds). Food Chem. 2004, 84, 367-374. 
44. Liu, X.; Jin, Q.; Liu, Y.; Huang, J.; Wang, X.; Mao, W.; Wang, S. Changes in volatile compounds of peanut oil during the roasting process for production of aromatic roasted peanut oil. J. Food Sci. 2011, 76, C404-C412.

45. Guillén, M.D.; Ibargoitia, M.L.; Sopelana, P.; Palencia, G. Components detected by headspacesolid phase microextraction in artisanal fresh goat's cheese smoked using prickly pear (Opuntia ficus indica). Le Lait 2004, 84, 385-397.

46. Soria, A.C.; González, M.; de Lorenzo, C.; Martínez-Castro, I.; Sanz, J. Estimation of the honeydew ration in honey samples from their physicochemical data and from their volatile composition obtained by SPME and GC-MS. J. Sci. Food Agric. 2005, 85, 817-824.

47. Wu, S.; Xu, T.; Akoh, C.C. Effect of roasting on the volatile constituents of Trichosanthes kirilowii seeds. J. Food Drug Anal. 2014, 22, 310-317.

48. Vázquez-Araújo, L.; Enguix, L.A.; Verdú, L.; García-García, E.; Carbonell-Barrachina, A.A. Investigation of aromatic compounds in toasted almonds used for the manufacture of turrón. Eur. Food Res. Technol. 2008, 227, 243-254.

49. Mexis, S.F.; Kontominas, M.G. Effect of oxygen absorber, nitrogen flushing, packaging material oxygen transmission rate and storage conditions on quality retention of raw whole unpeeled almond kernels (Prunus dulcis). Food Sci. Technol. 2010, 43, 1-11.

50. Krist, S.; Stuebiger, G.; Bail, S.; Unterweger, H. Analysis of volatile compounds and triacylglycerol composition of fatty seed oil gained from flax and false flax. Eur. J. Lipid Sci. Technol. 2006, 108, 48-60.

51. Amrani-Hemaimi, M.; Cerny, C.; Fay, L.B. Mechanisms of formation of alkylpyrazines in the Maillard reaction. J. Agric. Food Chem. 1995, 43, 2818-2822.

52. Krist, S.; Unterweger, H.; Bandion, F.; Buchbauer, G. Volatile compound analysis of SPME headspace and extract samples from roasted Italian chestnuts (Castanea sativa Mill.) using GC-MS. Eur. Food Res. Technol. 2004, 219, 470-473.

53. Ho, C.T.; Lee, M.H.; Chang, S.S. Isolation and identification of volatile compounds from roasted peanuts. J. Food Sci. 1981, 47, 127-133.

54. Agila, A.; Barringer, S. Effect of roasting conditions on color and volatile profile including HMF level in sweet almonds (Prunus dulcis). J. Food Sci. 2012, 77, 461-468.

55. Hsu, H.W.; Vavak, D.L.; Satterlee, L.D.; Miller, G.A. A multienzyme technique for estimating protein digestibility. J. Food Sci. 1977, 42, 1269-1273.

56. Bidlingmeyer, B.A.; Cohen, S.A.; Tarvin, T.L. Rapid analysis of amino acids using pre-column derivatization. J. Chomatogr. 1984, 336, 93-104.

57. Van den Dool, H.; Kratz, PD. A generalization of the retention index system including linear temperature programmed gas-liquid partition chromatography. J. Chromatogr. 1963, 11, 463-471.

58. National Institute for Standards and Technology. Available online: http://webbook.nist.gov/ chemistry/cas-ser.html (accessed on 23 October 2014).

Sample Availability: Samples of the compounds are available from the authors.

(C) 2015 by the authors; licensee MDPI, Basel, Switzerland. This article is an open access article distributed under the terms and conditions of the Creative Commons Attribution license (http://creativecommons.org/licenses/by/4.0/). 